\title{
Physiological Signal Processing for Individualized Anti-nociception Management During General Anesthesia: a Review
}

\author{
J. De jonckheere ${ }^{1}$, V. Bonhomme ${ }^{2}$, M. Jeanne ${ }^{1,3}$, E. Boselli, ${ }^{4}$ M. Gruenewald5, R. Logier ${ }^{1,6}$, P. Richebé ${ }^{7}$ \\ I INSERM CIC-IT 1403, Maison Régionale de la Recherche Clinique, CHRU de Lille, France \\ 2 University Department of Anesthesia and ICM, CHR Citadelle, Liege, Belgium \\ 3 Department of Anaesthesiology and Intensive Care, Hopitale Roger Salengro, CHRU de Lille, France \\ ${ }^{4}$ Department of Anaesthesiology and Intensive Care, Édouard Herriot Hospital, Hospices Civils de \\ Lyon, Lyon, France \\ 5 Department of Anaesthesiology and Intensive Care, University Hospital Schleswig Holstein Campus \\ Kiel, Kiel, Germany \\ 6 UDSL EA2694, Univeristé Lille Nord de France, Lille, France \\ 7 Department of Anaesthesiology, University of Montreal, Quebec, Canada
}

\begin{abstract}
Summary
Objective: The aim of this paper is to review existing technologies for the nociception / anti-nociception balance evaluation during surgery under general anesthesia.

Methods: General anesthesia combines the use of analgesic, hypnotic and muscle-relaxant drugs in order to obtain a correct level of patient non-responsiveness during surgery. During the last decade, great efforts have been deployed in order to find adequate ways to measure how anesthetic drugs affect a patient's response to surgical nociception. Nowadays, though some monitoring devices allow obtaining information about hypnosis and muscle relaxation, no gold standard exists for the nociception / anti-nociception balance evaluation. Articles from the PubMed literature search engine were reviewed. As this paper focused on surgery under general anesthesia, articles about nociception monitoring on conscious patients, in post-anesthesia care unit or in intensive care unit were not considered.

Results: In this article, we present a review of existing technologies for the nociception / anti-nociception balance evaluation, which is based in all cases on the analysis of the autonomous nervous system activity. Presented systems, based on sensors and physiological signals processing algorithms, allow studying the patients' reaction regarding anesthesia and surgery.

Conclusion: Some technological solutions for nociception / antinociception balance monitoring were described. Though presented devices could constitute efficient solutions for individualized anti-nociception management during general anesthesia, this review of current literature emphasizes the fact that the choice to use one or the other mainly relies on the clinical context and the general purpose of the monitoring.
\end{abstract}

\section{Keywords}

Physiologic monitoring, general anesthesia, nociception, tranducers, digital signal processing

Yearb Med Inform 2015;10:95-101

http://dx.doi.org/10.15265/IY-2015-004

Published online August 13, 2015

\section{Introduction}

General anesthesia combines the use of analgesic, hypnotic and muscle-relaxant drugs in order to render patients unconscious, disconnected from their environment and unresponsive to stimulation during surgery. During the last decade, great efforts have been deployed in order to find adequate ways to measure how anesthetic drugs affect a patient's response to surgically induced nociception. Nowadays, the real-time acquisition and analysis of a patient's physiological parameters provides an efficient way to continuously assess the response of drug administration in order to suppress nociception, thus making it possible for clinicians to personalize drug administration.

Depth of hypnotic component of anesthesia monitors have now been commercially available for many years and are used routinely: They record and process simplified electroencephalographic (EEG) signals and display the result in real-time, thus enabling anesthesiologists and nurse anesthetists to adjust hypnotic agent administration to reduce probability of intraoperative awareness [1] or discomfort as well as hypnotic overdose which is likely to be involved in postoperative delirium or cognitive dysfunction. EEG monitors then can calculate indexes related to the patient consciousness such as the bispectral index (BIS ${ }^{2}$, ASPECT Medical Systems, Newton, MA) or the state and response entropy index (SE and
RE, GE Healthcare Helsinki, Finland). The bispectral index constitutes a combination of several electroencephalographic parameters computed both in the time domain and in the frequency domain whereas the state and response entropy indexes are computed in the frequency domain and represent an evaluation of the dominated and the total parts of the spectrum. Both the technologies have proven their ability to evaluate consciousness of the patient under intravenous (propofol) or volatile agents (sevoflurane) anesthesia but may not be used under ketamine anesthesia [2].

Muscle relaxation monitors have also been used routinely for many years: They enable clinicians to precisely adapt muscle relaxation to the need of surgeons during the procedure, and to test for residual neuromuscular blockade before weaning the patient from ventilatory support.

Compared with other components of anesthesia, no gold standard exists for the assessment of nociception / anti-nociception (NAN) balance, despite several existing technical solutions, of which some have been commercialized during the last decade. Inadequate anti-nociception often results in blood pressure elevation or tachycardia. The converse, opioid overdose often results in bradycardia and low blood pressure episodes, and has been related to postoperative hyperalgesia [3]. Hence, the search for the optimal opioid administration is a difficult but important objective of modern anesthesia. 
In this article, we present a review of existing technologies for NAN balance evaluation, which is mainly based on the analysis of the Autonomous Nervous System (ANS) reactions to a changing environment during anesthesia and surgery.

\section{Pupillometry}

Pupillometry is a well-known method to study the ANS sympathetic activity. It consists in measuring the pupil diameter (PD) changes in response to different painful stimuli.

Larson et al. studied the effect of an electrical noxious stimulus on PD, blood pressure (BP) and heart rate (HR) in anesthetized patients [4]. They found that, even if stimulation significantly increased HR and BP, PD response to noxious stimulus was much more important. The authors concluded that PD was more sensitive to noxious stimulation than commonly used hemodynamic variables.

Constant et al tested PD, BP, HR and BIS response to skin incision in children [5]. They found that PD, BP and HR significantly increased after skin incision, whereas BIS didn't vary. Again, PD increase was more important than the increase of BP and HR. Moreover, opioid administration significantly reduced PD. The authors concluded that PD was more sensitive than commonly used variables to assess pain response in anesthetized children.

A few years ago, a French company, IDmed (IDMed, Marseille, France) developed a medical device based on the pupil response to noxious stimulus; the AlgiScan ${ }^{\circledR}$. Composed of an infrared 67 pictures/s camera, this system allows to monitor changes in PD and to study the pupillary reflex dilatation (PRD in \%) in response to a painful stimulus. This system also integrated electrodes and an electric stress generator allowing the performance of tetanic electrical stress in order to measure PRD response. As the system requires a direct access to the eye, its use is limited to an intermittent assessment of the NAN balance.

Isnardon et al. used this system to study the PRD response to a noxious stimulus during general anesthesia associated with an unilateral popliteal sciatic nerve block [6]. They tested the PD response to tetanic electrical stimulation applied 1) on the blocked leg, 2) on the non-blocked leg. They found that PD increased by $2 \%$ on the blocked leg whereas PD increased by $17 \%$ on the non-blocked leg. The authors concluded that PD allowed the investigation of the efficacy of a peripheral nerve block on blocking nociception. These results could also be considered as a proof of concept regarding PD ability to detect inadequate anti-nociception. However, PD could be affected in patients with ocular injury. Moreover, such a system does not allow continuous NAN balance monitoring.

\section{The Surgical Pleth Index (SPI)}

The Surgical Pleth Index (SPI) (GE Healthcare, Helsinski, Finland) is an index reflecting the ANS sympathetic activity. This index is computed from the signal issued from a photoplethysmographic sensor positioned on the finger. Such an optical sensor, composed of a light source and a photodetector (Figure 1), is able to measure volume changes in the microvascular

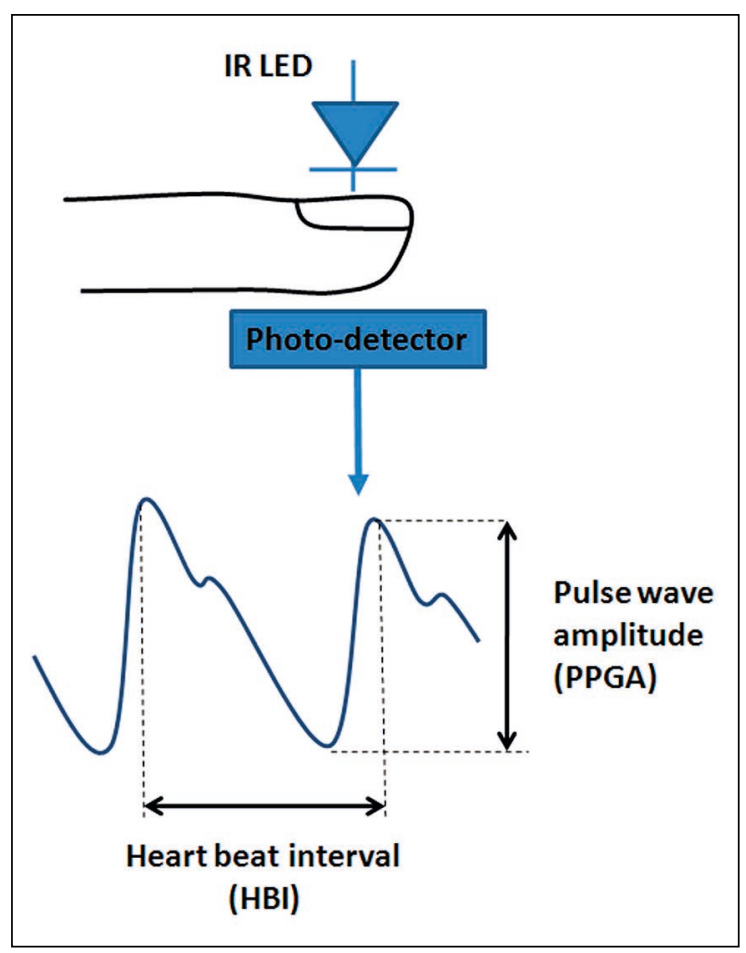

bed of tissues. The resulting microvascular pulse wave allows the computation of two parameters: the heart beat interval (HBI) and the PhotoPlethysmoGraphic pulse wave Amplitude (PPGA).

SPI (previously named the Surgical Stress Index, or SSI) was developed in order to provide a simple numerical measure of the level of surgical stress in an anesthetized patient [7]. To develop this index, authors studied the influence of a standard surgical stress on several physiological parameters with varying opioid concentrations. Studied parameters were; the normalized heart beat interval $\left(\mathrm{HBI}_{\text {norm }}\right)$, the normalized PhotoPlethysmoGraphic pulse wave Amplitude $\left(\mathrm{PPGA}_{\text {norm }}\right)$, the non-invasive systolic blood pressure (NIBP), the pulse transit time (PTT) and the Response entropy (RE). The authors defined a total surgical stress (TSS) index which depends both on stress predefined intensity (PreIntensity) and on opioid concentration $\left(\mathrm{Ce}_{\text {remi }}\right)$.

$$
\text { TSS = PreIntensity- }\left(\mathrm{Ce}_{\text {remi }} / 3 \mathrm{ng} / \mathrm{ml}\right) .
$$

The correlation of each parameter with TSS was measured in order to select the two best candidate variables regarding correlation, continuity and sensitivity to artifacts. After-

Fig. 1 Photoplethysmography and photopletysmographic waveform. An Infrared LED illuminates the skin and the photo-detector measures changes in light absorption due to blood flow. 
ward, PPGA ${ }_{\text {norm }}$ and $\mathrm{HBI}$ norm were selected to construct the model and SPI was defined as;

$$
\mathrm{SPI}=100-\left(0.7 * \mathrm{PPGA}_{\text {norm }}-\mathrm{HBI}_{\text {norm }}\right) \text {. }
$$

For clinical use, a SPI value close to 100 indicates a very high stress level whereas a SPI close to 0 indicates a very low stress level.

Struys et al. studied SPI, HR and RE ability to assess the balance between anti-nociception and stress level during general anesthesia [8]. They observed SPI, HR and $\mathrm{RE}$ response to a standard electrical tetanic stimulation under various opioid concentrations, and found that SPI correlates better with the opioid concentration than RE and HR. They concluded that SPI could be a useful measurement of the NAN balance.

In their study, Bonhomme et al. investigated SPI, HR and BP ability to assess the NAN balance during GA [9]. Even if the study design was very close to Struys' one, they found that, following noxious stimulation, SPI, HR and BP had comparable performances to predict the opioid concentration received. They also found that low intravascular volume and a history of treated chronic elevated blood pressure affected SPI, $\mathrm{HR}$ and BP interpretation.

Several studies showed the clinical benefit of using SPI for personalized anti-nociception management during general anesthesia. Chen et al. compared SPI-guided anti-nociception with standard clinical practice [10]. They found that SPI-guided opioid administration resulted in lower opioid consumption, better hemodynamic stability and lower incidence of unwanted events. With a similar clinical setting, Bergmann et al. found that SPI-guided opioid administration reduced both opioid and hypnotic drugs consumption, and resulted in faster recovery at the end of the procedure [11].

To conclude, SPI constitutes a non invasive continuous method for NAN monitoring. However, SPI could be affected by artifacts like vasoconstriction, hypovolemia or hypothermia.

\section{Skin Conductance}

Skin conductance (SC) is a well-known method to study the sympathetic nervous system activity. Through three electrodes positioned on the palmar surface of a patient's hand, it allows monitoring electrical conductance modulation due to the appearance/removal of sweat related to sympathetic activation/ inhibition. Storm et al. described algorithms designed to obtain information on the number of SC fluctuation (NSCF) and the amplitude of SC fluctuation (ASCF) [12] (Med-Storm Innovation, Oslo, Norway). To compute NSCF and ASCF, the system automatically detects the valleys and the peaks of the curves by analyzing the derivative of the wave. The amplitude is then computed as the difference between the SC value of the peak and the SC value of the previous valley. If this amplitude is higher than a predefined threshold $(0.02$ $\mu \mathrm{S})$, the peak is taken into account.

In a study aiming at evaluating the correlation between skin conductance and perioperative stress level [12], Storm et al. studied NSCF, ASCF, arterial catecholamines, HR, BIS and BP before, during and after general anesthesia for elective laparoscopic cholecystectomy. They found that NFSC and ASCF were positively correlated with BIS, BP, epinephrine level and norepinephrine level. Moreover, during tracheal intubation, NSCF showed the same response as the changes in norepinephrine levels whereas BIS did not vary. The authors concluded that NSCF could be a useful method to monitor perioperative stress.

In another study, Gjerstad et al. compared NSCF, RE and a clinical stress score during intubation and tetanic stimulation in patients under general anesthesia [13]. The clinical stress score was estimated in real time by observing the patient and was based on muscle movement, coughing, eye opening, sweating on the forehead, tears, face muscle reaction and systolic blood pressure (SBP) over $130 \mathrm{mmHg}$. They found that NSCF was positively correlated with the clinical stress score during intubation $\left(\mathrm{r}^{2}=0.73, \mathrm{p}<0.0005\right)$ whereas RE showed a less important correlation $\left(r^{2}=0.33, p=0.007\right)$. NSCF significantly increased after tetanic electrical stimulation $(p<0.001)$ whereas RE didn't vary. The authors concluded that NSFC was sensitive to noxious stimulation during GA. However, NSFC could be affected by hypothermia.

\section{Analgesia/Nociception Index (ANI)}

The Analgesia/Nociception Index (ANI) (Mdoloris Medical Systems, Lille, France) is a heart rate variability (HRV) based index. This technology continuously analyzes the influence of respiratory sinus arrhythmia (RSA) on HR, which leads to a measure of the relative parasympathetic tone. The method consists of detecting each $\mathrm{R}$ peak of an ECG signal in order to construct the $R R$ series (R-R interval evolution over time).

The RR series is then normalized and band pass filtered between 0.15 and $0.4 \mathrm{~Hz}$ in order to keep parasympathetic related variations only, which are mainly influenced by the respiration cycle.

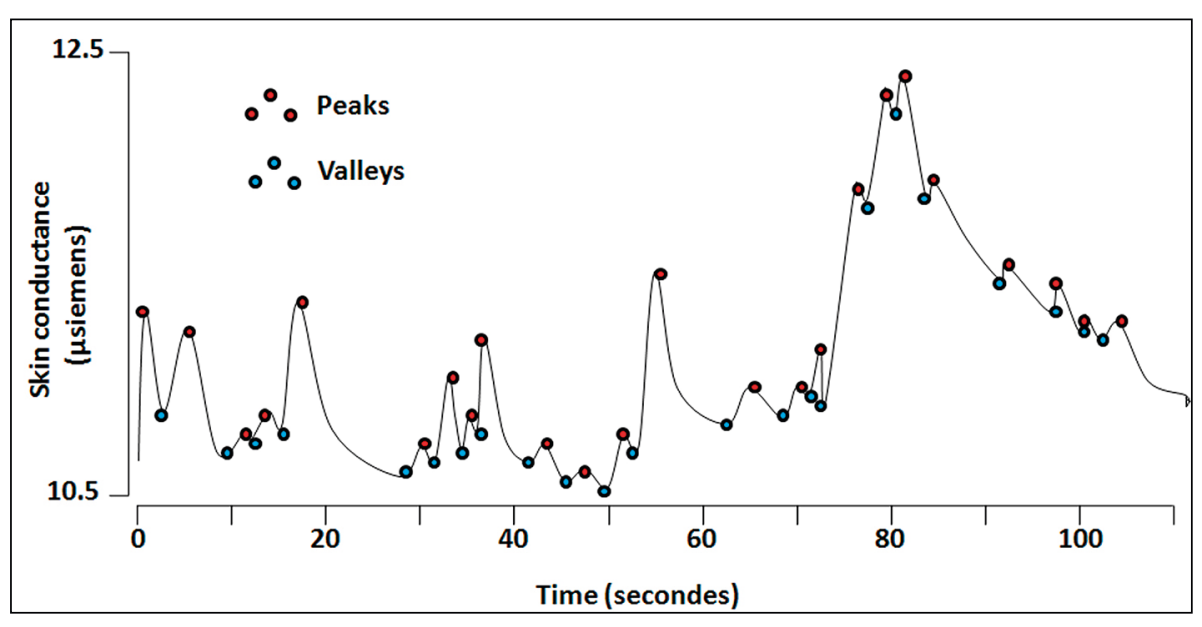

Fig. 2 Skin conductance level as a function of time. 


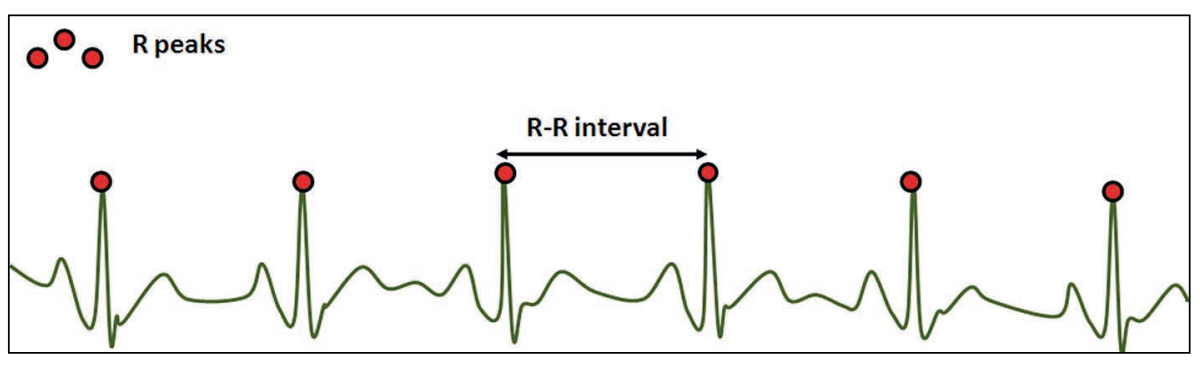

Fig. 3 ECG signal, R peak detection and R-R interval computation

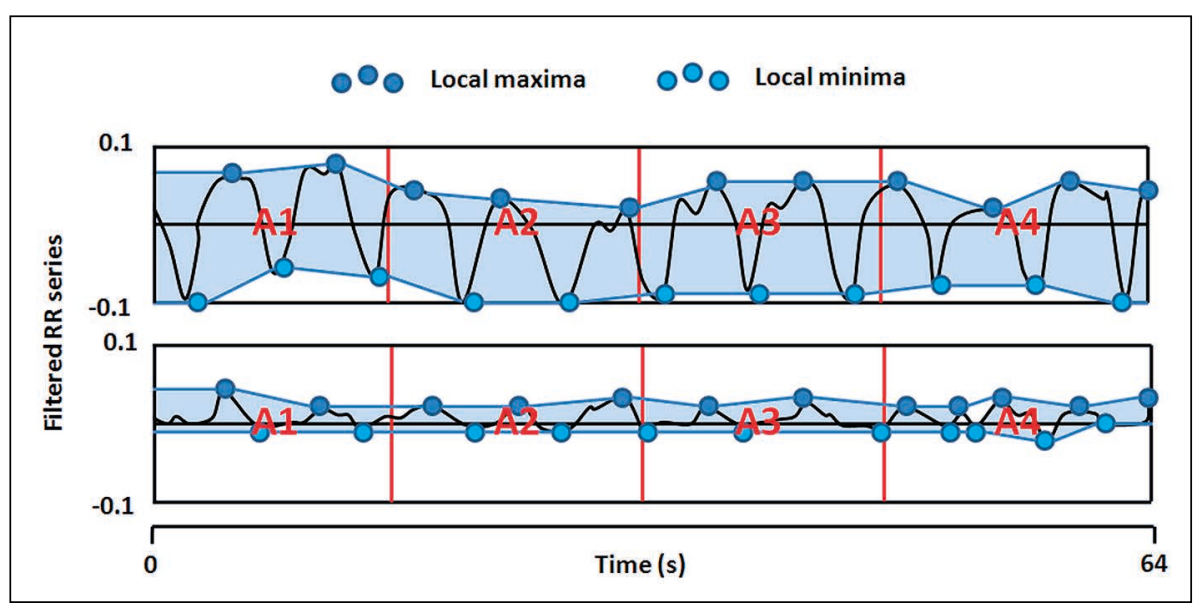

Fig. 4 Mean centered, normalized and band pass-filtered RR series in 2 different levels of NAN balance: 1) surgical stimulus in the case of adequate NAN balance (upper panel), 2) surgical stimulus in the case of inadequate anti-nociception (lower panel).

Local minima and maxima are then detected and the resulting envelope is divided into four subareas A1, A2, A3 and A4. AUCmin is defined as the minimum of the 4 subareas.

ANI is then computed as;

$$
\mathrm{ANI}=100 \times(\mathrm{a} \times \mathrm{AUCmin}+\mathrm{b}) / 12.8
$$

where $a=5.1$ and $b=1.2$ have been determined on a 200 patients dataset in order to keep coherence between the visual effect of respiratory influence on RR series and the quantitative measurement of ANI.

For clinical use, an ANI value close to 0 indicates a very high ANS response to stress which is a sign of low anti-nociception whereas an ANI close to 100 indicates a very low ANS response to stress.

Jeanne et al. studied ANI, HR and BP variations during general anesthesia [14] for laparoscopic abdominal surgery. Anesthesia induction was followed by a decrease in HR and BP, and an increase in ANI values. After the start of surgery, ANI decreased to 60 and decreased further to 50 after the stress stimulus (pneumoperitoneum inflation), while there were no significant changes in HR or BP. After completion of surgery, ANI returned to 90 . The authors concluded that ANI seems more sensitive than HR and BP to moderate noxious stimulation in anesthetized patients.

In another study, Jeanne et al. investigated how ANI could early detect hemodynamic reactivity defined as an increase in HR or BP by more than $20 \%$ [15]. A ROC curve analysis led to a threshold of 63 for hemodynamic reactivity early detection. The authors conclude that ANI values during general anesthesia are coherent with the evolution of the NAN balance and that maintaining ANI above 63 should decrease the rate of hemodynamic episodes, which needs further clinical validation. In another study, Boselli et al. also demonstrated ANI ability to predict hemodynamic reactivity on a larger population [16].
Boselli et al. also hypothesized that ANI could be used to predict postoperative pain felt in the post anesthesia care unit (PACU) [17]. The ANI was evaluated at the end of the surgical procedure, just before tracheal extubation and pain was self-reported by the patients 10 minutes after their arrival in the PACU using a 0-10 Numerical Rating Scale (NRS). A negative linear relationship was found between ANI measured before extubation and the NRS measured 10 minutes after the patient's arrival in PACU $\left(\mathrm{r}^{2}=\right.$ $0,33 ; p<0,01)$. An ANI threshold of 50 was identified as predictive of acute postoperative pain (NRS $>3$ ) with good sensitivity $(86 \%)$ and specificity $(86 \%)$. The authors concluded that ANI measured immediately before extubation is predictive of postoperative pain.

ANI constitutes a non invasive method for NAN balance continuous monitoring. However, ANI can be affected by several artifacts like arrhythmia, apnea or low respiratory frequency $(<9 \mathrm{cpm})$.

\section{CARDEAN}

The CARdiovascular DEpth of ANalgesia (CARDEAN, Alpha-2 Ltd, Lyon, France) index detects the occurrence of a minor elevation in blood pressure followed by minor tachycardia, which reflect the inhibition of the cardiac baroreflex. This index based on the evolution on BP and HR has retrospectively been designed in order to predict unexpected intraoperative movement in anesthetized and non-paralyzed patients [18]. According to the authors, in the case of adequate NAN balance, a minor elevation in blood pressure is followed by a decrease in $\mathrm{HR}$ (increase in RR interval, figure $5 \mathrm{~A}$ ) regulated by the cardiac baroreflex. The converse, when a minor elevation in blood pressure is followed by minor tachycardia (decrease in RR interval, figure $5 \mathrm{~B}$ ), the authors concluded in a cardiac baroreflex inhibition, which may indicate risk of intraoperative movement.

CARDEAN is a software that uses ECG and non-invasive continuous blood pressure sensors (Finapres medical systems, Amsterdam, Netherlands). In case of an increase in SBP between 2 and $5 \mathrm{mmHg}$, the CARDEAN algorithm analyses the 


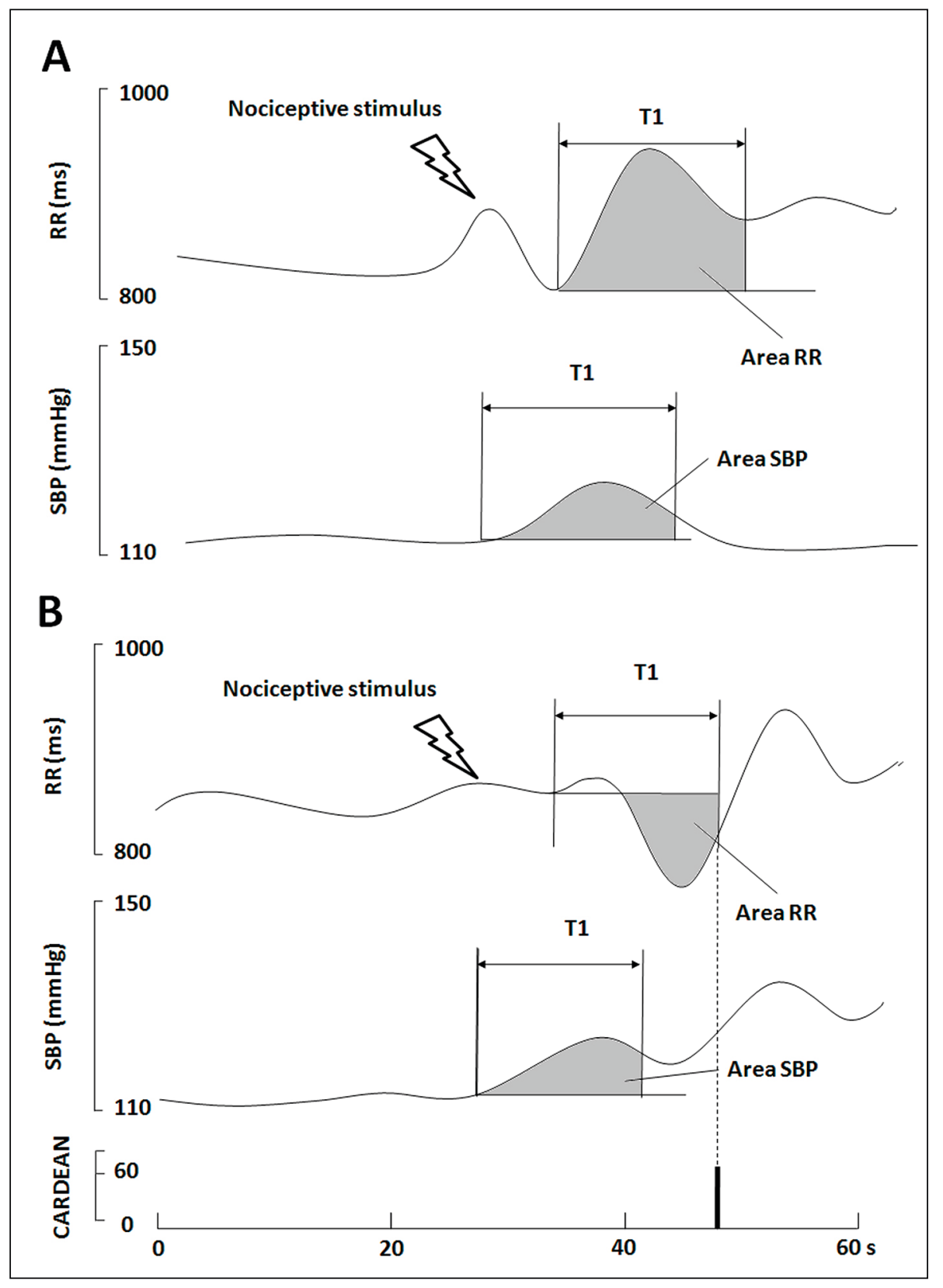

Fig. 5 RR and SPB series at 2 different levels of the NAN balance; A) surgical stimulus in case of adequate anti-nociception (upper panel), B) surgical stimulus in the case of inadequate anti-nociception (lower panel).

SBP and RR area under the curve as well as $\mathrm{RR}$ amplitudes in order to obtain an index related to the cardiac baroreflex activity. Thresholds values on SBP and RR area under the curve and RR amplitudes were determined to obtain the best specificity and sensibility regarding the occurrence of head, limb or finger movements. For clinical use, a CARDEAN higher than 60 is a sign of cardiac baroreflex inhibition.
In a first study, Martinez et al. investigated CARDEAN efficacy at reducing the occurrence of movement during surgery [19]. They compared CARDEAN-guided opioid administration with standard clinical practice. They concluded that CARDEAN-guided opioid administration significantly decrease the occurrence of intraoperative movements (by 51\%). However, the authors did not study the variations of CARDEAN under standardized noxious stimulus at different anti-nociception level neither the ability of CARDEAN to predict other sign of inadequate NAN balance.

Rossi et al. studied the CARDEAN response to skin incision at varying opioid concentration [20]. They measured the CARDEAN value before and after skin incision in two groups of patients: one group with a low opioid administration (remifentanil concentration of $2 \mathrm{ng} / \mathrm{mL}$ ) and one group with an important opioid administration (remifentanil concentration of $4 \mathrm{ng} / \mathrm{mL}$ ). After skin incision, the CARDEAN significantly increased in the first group whereas it did not vary in the second one. The CARDEAN was also predictive of tachycardia and elevated blood pressure (area under the ROC curve $=0.81$ ). The authors concluded that the CARDEAN was related to the adequacy of the NAN balance. However, CARDEAN could be affected by artifacts like vasoconstriction or hypovolemia.

\section{Wavelet Transform Cardiorespiratory Coherence}

The wavelet transform cardiorespiratory coherence (WTCRC) was developed to estimate the coupling between heart rate and respiration. The WTCRC applies a continuous wavelet transform to the HR time series and to a respiratory wave (for example expired $\mathrm{CO}_{2}$ ). Wavelet coefficients derived from the HR time series $\left(\mathrm{W}^{\mathrm{T}}\right)$ and respiratory time series $\left(\mathrm{W}^{\mathrm{R}}\right)$ are then used to compute the power spectrum of the HR time series $\left(\mathrm{W}^{\mathrm{TT}}\right)$, the power spectrum of the respiratory time series $\left(\mathrm{W}^{\mathrm{RR}}\right)$ and the cross power spectrum $\left(\mathrm{W}^{\mathrm{TR}}\right)$. A coherence estimator is defined as:

$$
\mathrm{C}=\left(\mathrm{W}^{\mathrm{TR}}\right) /\left(\mathrm{W}^{\mathrm{RR}} * \mathrm{~W}^{\mathrm{TT}}\right)
$$

WTCRC output vector corresponds to the coherence values at the known respiratory frequency.

As a proof of concept, Brouse et al. assessed the WTCRC ability to detect movements occurring during general anesthesia [21]. The WTCRC allowed detecting movements with a sensitivity of $95 \%$ for the minimum WTCRC value and $65 \%$ with the mean WTCRC. 
Brouse et al. studied WTCRC, BP and HR responses to 1) noxious stimulus (dental dam insertion) and 2) opioid injection [22]. They found that, even if stimulation significantly increased WTCRC, HR and $\mathrm{BP}, \mathrm{WTCRC}$ response to noxious stimulation was much more important than the response of HR and BP. The authors concluded that WTCRC was more sensitive than HR and BP to assess the balance between nociception and anti-nociception. However, WTCRC could be affected by several artifacts like arrhythmia or apnea.

\section{Multi-parameter Approach}

Ben-Israel et al. developed the nociception level (NoL) indexes that are based on the combination of multiple physiological parameters [23]. Based on a photoplethysmograpic sensor and skin conductance electrodes regrouped in single finger probe, the developed the PMD-100 $0^{\mathrm{TM}}$ (Medasense Biometrics Ltd, Ramat Yishai, Israel) device allows the extraction of parameters related to pulse-wave and skin conductance. The studied parameters were the photoplethysmograpic waveform amplitude (PPGA), HR, the skin conductance level (SCL) and the number of skin conductance fluctuation (NSCF). A high frequency heart rate variability (HRV-HF) was also computed as the power spectral density between 0.15 and $0.4 \mathrm{~Hz}$ using a Lomb-Scargle method.

The authors then defined two indexes regrouping all the studied parameters: $\mathrm{NoL}_{\text {linea }}$ which is based on a linear regression and $\mathrm{NoL}_{\text {non-linear }}$ which is based on a non-linear Random Forest regression.

In order to study indexes reactivity regarding the noxious stimuli during surgery under general anesthesia, the authors defined non-noxious periods as well as minor, moderate and major noxious stimulations. Besides, the authors defined a combined index of stimulus and anti-nociception (CISA) based both on the stimulus intensity (evaluated by clinicians) and the concentration of opioids, defined as follows:

$$
\mathrm{CISA}=\mathrm{Stim}_{\text {intensity }}-\beta^{*} \mathrm{Ce}_{\text {opioid }}+\gamma
$$

where $\beta=1 / 8$ and $\gamma=1.5$.
The authors demonstrated that the $\mathrm{NoL}_{\text {non-linear }}$ significantly increases after minor, moderate and major noxious stimulation while $\mathrm{NoL}_{\text {linear }}$ only increased after major and moderate noxious stimulation. The PPGA and HR significantly changed after major noxious stimuli whereas HRV-HF, SCL and NFSC did not vary. A ROC analysis showed that $\mathrm{NoL}_{\text {linear }}$ and $\mathrm{NoL}_{\text {non-linear }}$ better discriminate noxious and non-noxious events (area under the curve $=0.97$ ). The $\mathrm{NoL}_{\text {linear }}$ and $\mathrm{NoL}_{\text {non-linear }}$ were positively correlated with the CISA- $(r=0.77$ and 0.88 respectively). The authors concluded that the $\mathrm{NoL}_{\text {linear }}$ and $\mathrm{NoL}_{\text {non-linear }}$ constituted a better solution than individual parameters to evaluate the balance between nociception and anti-nociception.

\section{Discussion}

In this paper, we presented several existing technologies that allow the evaluation of the NAN balance during general anesthesia. Even if these technologies have proven their ability to evaluate the ANS response to noxious stimulation, the choice to use one or the other mainly relies on the clinical context and the general purpose of the monitoring: intermittent versus continuous, routine versus clinical research, etc. Does the clinician want to limit the opioid administration or the postoperative pain? Does he want to limit patient movements and/or hemodynamic reactivity?

Moreover, even if some technologies are already commercially available (SPI, SC, PRD, ANI), others are still under development and therefore not marketed (CARDEAN, WTCRC, NoL).

Several clinical studies have been performed to compare the different technologies in various clinical settings. Sabourdin et al. studied the ANI and skin conductance response to noxious stimulation under varying opioid flow rates in a pediatric population [24]. The ANI decreased significantly after each noxious stimulation for all opioid flow rates, while skin conductance, $\mathrm{HR}$ and $\mathrm{BP}$ were not modified. The authors concluded that, in their clinical setting, the ANI might be a more sensitive tool to evaluate the NAN balance than HR, BP or skin conductance.
Ledowski et al. studied the relationship between SPI, AFSC and NFSC, and the stress hormones level [25]. The SPI, NFSC, HR and BP were recorded and blood samples (plasma level of adrenaline, noradrenaline, cortisol...) were withdrawn during GA. The authors showed that neither SPI nor NFSC reflected stress hormones changes accurately. However, SPI significantly decreased after opioid administration whereas NFSC did not vary.

The PRD evaluated by the AlgiScan ${ }^{\circledR}$ was compared to the ANI to assess the effectiveness of regional anesthesia in children under GA [26]. The pupillary diameter and the ANI were recorded immediately before skin incision, and every 30 seconds during 2 minutes. The authors defined regional anesthesia failure by a $10 \%$ increase in HR within 2 minutes following the skin incision. The PRD and the ANI significantly changed after skin incision. Failure of regional anesthesia was predicted by PRD and ANI with reasonable areas under the ROC curves (respectively 0.671 and 0.747 ). The authors concluded that both ANI and PRD vary with skin incision and that they could represent efficient tools to assess the effectiveness of regional anesthesia.

Gruenewald et al. studied the ANI, SPI, BIS, HR and BP response to painful stimulation under various levels of anti-nociception [27]. They observed that BIS, HR and BP did not change after noxious stimulation whereas SPI and ANI varied after each stimulation: They also noted that higher opioid rates resulted in higher ANI and lower HR and BP values whereas SPI and BIS were not influenced. The authors concluded that both ANI and SPI could detect possible inadequate NAN balance.

\section{Conclusion}

More than identifying the "best" monitor of the NAN balance, this review of current literature emphasizes the fact that individual characteristics of each monitoring device, and hence the physiological understanding it provides, may be of importance when choosing a monitor for anyone's practice. In our view, it is of most importance that the user 
understands how a given monitor is working, in order to adequately use the information it provides and eventually adapt the administration of anesthetic drugs. When looking into the clinical designs used for monitoring validation, absolute values seem more or less questionable in all existing devices because of the lack of a gold standard. However, dynamic values may be of better clinical value because they give an insight, over a short period of time, of how an individual's ANS is reacting to a somewhat reproducible nociceptive stimulus, e.g. electric stimulation or skin incision.

\section{Conflict of interest}

De Jonckheere J, Jeanne M and Logier R are scientific advisers for MDoloris Medical Systems, and own shares of MDoloris Medical Systems.

\section{References}

1. Myles PS, Leslie K, McNeil J, Forbes A, Chan MT. Bispectral index monitoring to prevent awarness during anaesthesia : the B-Aware randomised controlled trial. Lancet 2004 May 29;363(9423):1757-63.

2. Bein B. Entropy. Best practice \& research clinical anesthesiology 2006. 20(1):101-109.

3. Joly V, Richebé P, Guignard B, Fletcher D, Maurette $\mathrm{P}$, Sessler DI, et al. Remifentanil-induced postoperative hyperalgesia and its prevention with smalldose ketamine. Anesthesiology 2005;103:147-55.

4. Larson MD, Sessler DI, Washington DE, Merrifield BR, Hynson JA, McGuire J. Pupillary response to noxious stimulation during isoflurane and propofol anesthesia. Anesth Analg 1993;76:1072-8.

5. Constant I, Nghe, MC, Boudet L, Berniere J, Schrayer S, Seeman R, et al. Reflex pupillary dilatation in response to skin incision and alfentanil in children anaesthetized with sevoflurane: a more sensitive measure of noxious stimulation than the commonly used variable. Br J Anaesth 2006;96: 614-9.

6. Isnardon S, Vinclair M, Genty C, Hebrard A, Albaladejo P, Payen J. Pupillometry to detect pain response during general anaesthesia following unilateral popliteal nerve block: a prospective, observational study. Eur J Anaesth 2013;30:429-34.

7. Huiku M, Uutela K, van Gils M, Korhonen I, Kymäläinen M, Meriläinen $\mathrm{P}$, et al. Assessment of surgical stress during general anaesthesia. Br J Anaesth 2007;98:447-55.

8. Struys MM, Vanpeteghem C, Huiku M, Uutela $\mathrm{K}$, Blyaert NB, Mortier EP. Changes in a surgical stress index in response to standardized pain stimuli during propofol-remifentanil infusion. $\mathrm{Br}$ J Anaesth 2007;99:359-67.

9. Bonhomme V, Uutela K, Hans G, Maquoi I, Born JD, Brichant JF, et al. Comparison of the surgical Pleth Index ${ }^{\mathrm{TM}}$ with haemodynamic variables to assess nociception-anti-nociception balance during general anaesthesia. Br J Anaesth 2011;106:101-11.

10. Chen X, Thee C, Gruenewald M, Wnent J, Illies C, Hoecker J, et al. Comparison of surgical stress index-guided analgesia with standard clinical practice during routine general anesthesia: a pilot study. Anesthesiology 2010;112:1175-83.

11. Bergmann I(1), Göhner A, Crozier TA, Hesjedal B, Wiese CH, Popov A et al. Surgical pleth index-guided remifentanil administration reduces remifentanil and propofol consumption and shortens recovery times in outpatient anaesthesia. Br J Anaesth 2013 Apr;1 10(4):622-8.

12. Storm H, Myre K, Rostrup M, Stokland O, Lien D, Raeder JC. Skin conductance correlates with perioperative stress. Acta Anaesthesiol Scand 2002:46:887-95.

13. Gjerstad AC, Storm H, Hagen R, Huiki M, Qvigstad E, Raeder J. Comparison of skin conductance with entropy during intubation, tetanic stimulation and emergence from general anaesthesia. Acta Anaesthesiol Scand 2007;51:8-15.

14. Jeanne M, Clement C, De Jonckheere J, Logier $\mathrm{R}$, Tavernier B. Variations of the analgesia nociception index during general anaesthesia for laparoscopic abdominal surgery. J Clin Monit Comput 2012;26:289-94

15. Jeanne M, Delecroix M, De Jonckheere J, Keribedj A, Logier R, Tavernier B. Variations of the Analgesia Nociception Index during propofol anesthesia for total knee replacement. Clin J Pain 2014 Dec;30(12):1084-8

16. Boselli E, Bouvet L, Begou G, Torkmani S, Allaouchiche B. Prediction of haemodynamic reactivity during total intravenous anaesthesia for suspension laryngoscopy using Analgesia/Nociception Index (ANI): a prospective observational study. Minerva Anestesiol 2015 Mar;81(3):288-97.

17. Boselli E, Bouvet L, Bégou G, Dabouz R, Davidson J, Deloste JY, et al. Prediction of immediate postoperative pain using the analgesia/nociception index: a prospective observational study. $\mathrm{Br} \mathrm{J}$ Anaesth 2014;112:715-21.

18. Cividjian A, Martinez JY, Combourieu E, Precloux P, Beraud AM, Rochette Y, et al. Beat by beat cardiovascular index to predict unexpected intraoperative movement in anesthetized unparalyzed patients: a retrospective study J Clin Monit Comput 2007;21:91-101.
19. Martinez JY, Wey PF, Lions C, Cividjian A, Rabilloud M, Bissery A, et al. A beat by beat cardiovascular index, CARDEAN: A prospective randomized assessment of its utility for the reduction of movement during colonoscopy. Anesthes Analg 2010;110:765-72.

20. Rossi M. Cividjian A, Fevre MC, Oddoux ME, Carcey J, Halle C, et al. A beat by beat, on-line, cardiovascular index, CARDEAN, to assess circulatory response to surgery: a randomized clinical trial during spine surgery. J Clin Monit Comput 2012;26:441-9.

21. Brouse CJ, Karlen W, Myers D, Cooke E, Stinson J, Lim J, et al. Wavelet transform cardiorespiratory coherence detects patient movement during general anesthesia. Conf Proc IEEE Eng Med Biol Soc. 2011;2011:6114-7.

22. Brouse CJ, Karlen W, Dumont GA, Myers D, Cooke E, Stinson J, et al. Monitoring nociception during generale anesthesia with cardiorespiratory coherence. J Clin Monit Comput 2013;27:551-60.

23. Ben-Israel N, Kliger M, Zuckerman G, Katz Y, Edry R. Monitoring the nociception level: a multi-parameter approach. J Clin Monit Comput 2013;27:659-68.

24. Sabourdin N, Arnaout M, Louvet N, Guye ML, Piana F, Constant I. Pain monitoring in anesthetized children: first assessment of skin conductance and analgesia nociception index at different infusion rates of remifentanil. PaediatrAnaesth 2013;23:149-55.

25. Ledowski T, Pascoe E, Ang B, Schmarbeck T, Clarke MW, Fuller C, et al. Monitoring of intra-operative nociception: skin conductance and surgical stress index versus stress hormone plasma level. Anaesthesia 2010;65:1001-6.

26. Migeon A, Desgranges FP, Chassard D, Blaise BJ, De Queiroz M, StewartA, et al. Pupillary reflex dilatation and analgesia nociception index monitoring to assess the effectiveness of regional anesthesia in children anesthetised with sevoflurane. Paediatr Anaesth 2013;23:1160-5.

27. Gruenewald M, Ilies C, Herz J, Schoenherr T, Fudickar A, Hocker J, et al. Influence of nociceptive stimulation on analgesia nociception index (ANI) during propofol remifentanil anaesthesia. Br J Anaesth 2013;110:1024-30
Correspondence to:
Julien De jonckheere
INSERM CIC-IT 1403
CHRU de Lille
Maison Régionale de la Recherche Clinique
6 rue du professeur Laguesse
59037 Lille Cedex, France
Tel: +33-(0)3-20-44-67-54
E-mail: Julien.dejonckheere@chru-lille.fr or
i.dejonckheere@hotmail.fr 